\title{
17. Big Data in der personalisierten Medizin - ethische Herausforderungen und Lösungsansätze
}

Dieses Kapitel beleuchtet die Voraussetzungen, die Chancen, beobachtbaren Entwicklungen und ethischen Herausforderungen einer Medizin, die als personalisierte Medizin, Systemmedizin oder Präzisionsmedizin bezeichnet wird und wesentlich auf der Idee basiert, alle gesundheitsrelevanten Daten einer Person so zu erfassen, dass Prävention, Diagnose und Therapie stärker auf die biologischen Eigenschaften der Erkrankung einer Person zugeschnitten werden können. Hierfür werden zunächst die zentralen Begriffe „Big Data“ und „personalisierte Medizin“, sowie ihre Verwendung und Begriffsgeschichte eingeführt (Abschnitt 17.1 und 17.2). Gerade weil einerseits Daten und Wissen aus beiden Handlungsfeldern - Forschung und Gesundheitsversorgung für eine datenintensive und personalisierte Medizin systematisch zusammengeführt werden sollen und andererseits unterschiedliche Prinzipien in der ärztlichen Ethik und Forschungsethik handlungsleitend sind, stellen sich hier wichtige ethische Fragen (Abschnitt 17.3): Etwa nach dem Umgang mit Zufallsbefunden, nach der Verantwortung von Forscher*innen für den Schutz der Privatsphäre der Patient*innen oder die Möglichkeiten einer informierten Einwilligung.

Deutlich wird hierbei, dass, neben den individuellen Kontrollmöglichkeiten über Daten, die Anforderungen an eine transparente und rechenschaftspflichtige Daten-Governance, also der Lenkung und Aufsicht der Erhebung und Nutzung von Daten, immer wichtiger werden (Abschnitt 17.4). Diese wird zum einen im Rückgriff auf das Konzept einer solidarischen Datennutzung mit Blick auf notwendige Regeln der Daten-Governance ausgeführt (Abschnitt 17.4.1). Zum anderen wird im Abschnitt 17.4.2 gefragt, inwiefern es eine moralische Pflicht geben kann, seine klinischen Daten für die Forschung zur Verfügung zu stellen. Verschiedene Möglichkeiten, persönliche Daten für die Forschung freizugeben, werden in Abschnitt 17.4.3 vorgestellt - insbesondere am Beispiel der sogenannten „Datenspende“. Zuletzt (Abschnitt 17.5) werden die Konzepte des Empowerments und der Partizipation, die im Zusammenhang mit datenintensiver 
Forschung immer häufiger diskutiert werden, kritisch in den Blick genommen. Statt einer inflationären Anwendung dieser Begriffe auf alle Praktiken, in denen Patient*innen aktiv etwas beitragen, legen wir dar, wie Formen von Patient*innenbeteiligung gefördert werden können, die den Handlungsraum der Menschen sinnvoll erweitern.

\subsection{Was ist Big Data?}

„Big Data“ ist ein Begriff, der häufig in einem Atemzug mit personalisierter Medizin genannt wird. Allerdings bedeutet dieser Begriff in unterschiedlichen Disziplinen und Praxiskontexten durchaus Verschiedenes; eine allgemeingültige Definition von Big Data gibt es nicht. Grob gesprochen lassen sich folgende Zugänge unterscheiden (siehe auch BEK, 2019):

Ein wichtiger Ansatz sieht Big Data als inkrementales Phänomen (BEK, 2019: 23). Diese Definition geht davon aus, dass Big-Data-Praktiken nicht unbedingt prinzipiell anders als bisheriges Arbeiten mit größeren Datensätzen sind, sondern dass es sich um eine graduelle Veränderung in vielen Hinsichten handelt: nämlich, dass es sich um Datensätze handelt, die besonders groß sind (Volume), sehr schnell analysiert werden (Velocity) oder aus unterschiedlichen Quellen kommen bzw. unterschiedliche Formate haben (Variety). Zusätzlich zu diesen „drei V's“ haben unterschiedliche Autor*innen noch andere Charakteristika vorgeschlagen, wie Wahrheitsgehalt/Qualität (Veracity), Wert (Value) oder Komplexität (Complexity) (eine Übersicht bieten z. B. Favaretto et al., 2020; Laney, 2011). Kitchin und McArdle (2016) kritisieren, dass viele der in diesem Kontext diskutierten Eigenschaften Herausforderungen bezeichnen, die sich im Kontext der Arbeit mit Big Data ergeben, die jedoch keine seinsbestimmenden Charakteristika von Big Data seien. Eng verbunden mit dem vorangegangenen Ansatz subsumieren andere Definitionen jene Datensätze unter Big Data, deren Speicherung und Verarbeitung mit vorhandenen Mitteln nicht bewerkstelligt werden kann. (Favaretto et al., 2020: 8/12; BEK, 2019: 23; Big Data als computationales Problem).

Wiederum andere Zugänge verstehen unter Big Data nicht nur Datensätze, sondern neue methodologische Zugänge - insbesondere jene, die „hypothesenfrei“ in großen Datensätzen nach Korrelationen suchen (Cukier/Mayer-Schönberger, 2013). Damit unterscheiden sie sich von Zugängen, die die Datenanalyse mit einer bestehenden Arbeitshypothese oder einer konkreten Forschungsfrage beginnen (BEK, 2019: 24; ElSayed/Prainsack, 2021). Wenn Korrelationen erkannt werden, dann kann in weiterführender Forschungsarbeit untersucht werden, welche Kausalzusammenhänge - wenn überhaupt - ihnen unterliegen; dies bedeutet, die Arbeitshypothese ergibt sich erst aus den Daten. Gerade in den letzten Jahren wird Big Data nicht nur als Datensätze großer 
Größe, Heterogenität und Komplexität verstanden, sondern auch als bestimmte Zielsetzung definiert: nämlich neue Assoziationen zu entdecken, die hypothesengenerierend sind (Hulsen et al., 2019).

Manche Ansätze gehen sogar so weit, nur jene Datensätze als Big Data zu bezeichnen, die einen Organismus oder ein Phänomen in seiner Gesamtheit abbilden (BEK, 2019: 24). „Digital twins“-Ansätze - also die Idee, dass man etwa Organe als Computersimulation abbildet, um dann Prozesse, wie etwa die Wirkung von Medikamenten, im digitalen Doppelgänger statt im lebenden Organismus zu simulieren - gehen in diese Richtung (siehe z. B. Bruynseels et al., 2018). Weil aber gleichzeitig immer mehr Aufmerksamkeit auf jene Aspekte gelenkt wird, die solche Ansätze eines technowissenschaftlichen Holismus (Vogt et al., 2016) - also eine Ganzheitlichkeit, die den Körper in einzelne Datenpunkte zerteilt und dann wieder zusammensetzt - notwendigerweise vermissen lassen, hat sich dieser Zugang zur Definition von Big Data nicht durchgesetzt.

Dafür werden derzeit Zugänge immer bedeutender, die das Phänomen Big Data noch weiter fassen, nämlich als technosoziale Praxis im Kontext digitaler Transformationsprozesse (technosoziales Phänomen; siehe BEK, 2019: 24). Hier meint Big Data also nicht bloß große Datensätze und die dazugehörigen materiellen Technologien, sondern auch die datenmäßige Erfassung immer weiterer Teile unserer Körper und unseres persönlichen und sozialen Lebens („Datafizierung“) (Cukier/ Mayer-Schönberger, 2013; Simon/ Rieder, 2017).

\subsection{Was hat Big Data mit personalisierter Medizin zu tun?}

Wenn man Big Data nicht nur, in einem engen Sinn, als besonders große Datensätze versteht, sondern als eine immer weiter voranschreitende Erfassung von Daten und Informationen über unterschiedliche Aspekte biologischer Prozesse im Individuum, Lebensstile und der Einflussfaktoren unserer nichtmenschlichen Umwelt, dann ist personalisierte Medizin ohne Big Data nicht denkbar. Die Idee, Prävention, Diagnose und Therapie stärker auf die individuellen biologischen Eigenschaften von Patient*innen zuzuschneiden, benötigt als Vorbedingung, dass es die Information über diese individuellen Eigenschaften überhaupt gibt. Dazu ist es nicht nur notwendig, Daten, die im Gesundheitswesen routinemäßig generiert werden, auszuwerten, sondern auch Informationen über den Lebensstil, oder epidemiologische und Umweltdaten (Prainsack, 2017a). Ein Bericht der U.S. National Academies of Sciences aus dem Jahr 2011 (NAS) formulierte erstmals, was später zu einer paradigmatischen Vision der personalisierten und Präzisionsmedizin wurde. Der Bericht schlug vor, traditionelle, symptombasierte Krankheitsklassifikationen durch eine neue Klassifikation zu ersetzen, die auf daten- 
intensiven Charakterisierungen individueller Patient*innen in unterschiedlichen Phasen der Krankheit basiert. Der Bericht verwendet die Analogie von „Google Maps“: so wie die Funktion Google Maps, die aus unterschiedlichen Schichten von Datenarten besteht, die übereinander gelegt eine Karte ergeben, die sich zur Navigation eignet und auch eine zeitliche Dimension miterfasst, sollten in dieser Vision auch „Karten“ menschlicher Gesundheitsdaten aussehen. Die Vision des Berichts ist, dass diese Karten sowohl den Bedürfnissen medizinischer Praxis als auch der Forschung dienen könnten (NAS, 2011: 17).

In einem solchen System patientenzentrierter Daten mögen molekulare Daten in manchen Kontexten noch die erste Geige spielen, aber sie ist eindeutig Teil eines größeren Orchesters.

In den späten 1990er- und frühen 2000er-Jahren bestand das Versprechen der personalisierten Medizin darin, die medikamentösen Behandlungen auf die individuellen genetischen Merkmale der Patient*innen abzustimmen.

\subsubsection{Frühe Kritik und heutiger Stand der personalisierten Medizin}

Die Begriffe „personalisierte“ oder ,individualisierte“ Medizin haben eine breite Kritik erfahren. Kritisiert wurde etwa, dass „personalisiert“ eine personen- und patientenzentrierte Behandlung suggeriere, es aber vielmehr um eine an Biomarkern ausgerichtete Behandlung ginge. Biomarker sind dabei beispielsweise Blutwerte oder andere biologisch messbare Parameter. Auch handele es sich um eine Gruppeneinteilung nach Biomarkern und eben nicht um eine „individualisierte“ Therapie im Sinne einer maßgeschneiderten Behandlung, die nur für ein Individuum hergestellt wird. In Reaktion auf die breite Kritik, die die Bezeichnung „personalisierte Medizin“ erfahren hat, wählte der US-amerikanische Nationale Forschungsrat (National Research Council) den Ausdruck „Präzisionsmedizin“ bewusst als Korrektur - oder Klarstellungsbegriff.

Mittlerweile ist beispielsweise in der Krebsmedizin die molekulare Analyse der Tumorzellen Voraussetzung für die Wahl der besten Behandlungsstrategie (siehe Fehse, Kap. 6). Die Behandlung von Patient*innen mit Lungenkrebs ist ein paradigmatisches Beispiel für die Veränderung durch die Präzisionsmedizin. Hat man vor 20 Jahren bei Lungenkrebs noch alle Patient*innen mit Chemotherapie behandelt und nur zwei verschiedene Untertypen unterschieden, so ermöglicht die Kenntnis des molekularen Profils der Tumorzellen bei vielen Patient*innen heute eine gezielte, Biomarker-adaptierte Behandlung. Nimmt man die drei häufigsten Treibermutationen zusammen, können heute etwa ein Drittel der Patient*innen mit nicht-kleinzelligem Lungenkarzinom mit Medikamenten behandelt werden, die in den spezifischen Stoffwechselweg eingreifen, 
der bei ihrem Tumor das Wachstum bestimmt. Für diesen Teil der Patient*innen bedeutet die Präzisionsmedizin einen deutlichen Zugewinn an Lebenszeit und Lebensqualität.

Für die Ärzt*innen bedeutet dies zunächst einmal einen enormen Wissensgewinn und eine Diversifizierung der Therapie in Abhängigkeit von der molekularen Charakterisierung der Tumorzellen - den es zunächst einmal zu meistern gilt und der sich auch in beinahe allen unterschiedlichen Krebsarten rasant erweitert. Auch wenn diese Medikamente nicht „maßgeschneidert“ werden für jede/n einzelne/n Patient*in, richten sich die Therapiesequenzen zunehmend nach dem biologischen Tumorgeschehen; wenn beispielsweise in einem zweiten oder dritten Schritt bereits Medikamente gegen spezifische Resistenzmutationen eingesetzt werden, sodass die jeweilige Therapieabfolge immer stärker individualisiert wird. Eine tatsächlich maßgeschneiderte Therapie wurde 2017 mit der Car-T-Zell-Behandlung für Kinder und Jugendliche mit schweren Blutkrebserkrankungen zugelassen: Die Therapie ist auf jede/n Patient*in individuell zugeschnitten und besteht aus einem lebenden Arzneimittel aus patientenindividuellen Zellen, die dem Immunsystem des/der Patient*in beibringen, spezifisch gegen die eigenen Krebszellen vorzugehen (siehe Fehse, Kap. 6). Die Vision einer individualisierten Medizin ist für diese Patient*innengruppe realisiert. Für viele andere Erkrankungen ist der Weg dahin noch weit.

\subsubsection{Zukunft der datenintensiven Medizin - Systemmedizin und Präzisionsmedizin}

Eine der wichtigen Lehren aus dem Humangenomprojekt in den frühen 2000er-Jahren war es, dass die menschlichen Genome weitaus komplexer und variabler sind, als Wissenschaftler*innen angenommen hatten, und dass die DNA-Sequenz allein nur sehr wenig bestimmt (siehe Rheinberger/Müller-Wille, Kap. 11). Dies ist einer der Gründe dafür, dass sich die personalisierte Medizin auf andere Datentypen wie etwa die Proteine, die produziert werden, Daten zum Stoffwechsel oder auch zu gesundheitsrelevantem Verhalten und umfassendere Überwachungsmethoden ausgedehnt hat. Die Vision ist weiterhin die einer Medizin, die alle relevanten Daten eines/einer Patient*in und das für ihre/seine Situation relevante Wissen aus Forschung und Routineversorgung in der Diagnose sowie in Therapie- und Präventionsentscheidungen in Erwägung zieht. Dieser Zugang wird aufgrund seiner Grundlagen in der Systembiologie manchmal auch mit dem Begriff „Systemmedizin“ beschrieben. Die Systemmedizin ist eine Medizin, die zielgerichtete Präventions- und Therapiemaßnahmen ermöglicht durch die bioinformatische Verarbeitung und Modellierung großer Datenmengen aus verschiedenen 
Quellen (z. B. klinische, epidemiologische oder eben die oben beschriebenen molekularen und genetischen Daten) (Schleidgen et al., 2017).

Relevant hierfür können alle Informationen werden, die Einflussfaktoren auf Gesundheit oder Krankheit sind. Die Zukunft liegt für Befürworter*innen einer solchen umfassenden, datengetriebenen Medizin - ob sie nun personalisierte oder „Präzisions-“, oder Systemmedizin genannt wird - in der Verwendung verschiedener Datensätze aus klinischen, persönlichen, öffentlichen und kommerziellen Bereichen, einschließlich Informationen zum Lebensstil, Daten aus öffentlichen Archiven, von tragbaren Sensoren und Postings in sozialen Medien. Dennis Ausiello und Scott Lipnick argumentieren etwa, es sei entscheidend, auch Daten und Informationen darüber zu haben, was zwischen den Arztbesuchen passiert - also auch über Phasen, in denen Menschen keine akuten gesundheitlichen Probleme haben (Ausiello/Lipnick, 2014 und 2015). Die nächste große Herausforderung in der Medizin bestehe darin, „Wohlbefinden und Krankheit auf eine kontinuierliche Art und Weise zu quantifizieren, während unsere Patienten ihr tägliches Leben führen“ (Ausiello/Lipnick, 2015). ${ }^{1}$ Mobiltelefone, tragbare Sensoren und andere Messgeräte sollten verwendet werden, um „die Erfassung von Messungen für den Einzelnen unauffälliger zu machen“ (Ausiello/Lipnick, 2014). Ein weiterer Vorteil, den diese Autoren im Ansatz der „kontinuierlichen Gesundheit“ („continuous health") sehen, ist, dass Daten von solchen Geräten direkt an medizinische Teams und Pflegekräfte gesendet werden können, um die Datenerfassung „vollständiger“ zu machen. Das Ziel ist es dabei, longitudinale Datensätze zu erstellen, die eine Reihe von relevanten Parametern über die Patient*innen während ihres gesamten Lebensverlaufs charakterisieren. Auf diese Weise werden Patient*innen zu ihren eigenen „Kontrollgruppen“ (Prainsack, 2017a).

\subsection{Ethische Herausforderungen an der Schnittstelle von Forschung und Krankenversorgung}

In der Medizin zeichnen sich mehrere vielversprechende Anwendungsfelder von Big Data in Forschung und Versorgung ab, gerade auch an der Schnittstelle zwischen den Bereichen der schnellen Translation von Wissen aus der Forschung in die Versorgung. Der Erfolg der Forschung in der Präzisionsmedizin beruht beispielsweise weitgehend auf der Integration von Forschungsdaten und realen Patient*innendaten. In der Krankenversorgung könnten - wenn die rechtlichen Voraussetzungen dafür geschaffen werden - sogenannte lernende Gesundheitssysteme Daten etwa zum Keimspektrum,

1 Übersetzungen ins Deutsche stammen, wenn nicht anders angegeben, von den Autorinnen. 
zu Infektionserregern und Antibiotikaresistenzen in einem Krankenhaus, einer Stadt oder einer Region in Echtzeit monitorieren und diese Information für Hygienemaßnahmen und die Antibiotikawahl zur Verfügung stellen; Krankenkassen könnten über den Abgleich von Abrechnungsdaten Rückmeldung an die Hausärzt*innen oder die Patient*innen zu wichtigen Medikamenteninteraktionen geben, wenn das in Zukunft gesetzlich erlaubt wird; die Verträglichkeit und Wirksamkeit neu zugelassener Medikamente könnte nach Markteinführung eines Medikaments leichter überwacht werden.

Gerade wegen des erwünschten schnellen Austauschs von Wissen und Information an der Schnittstelle zwischen Forschung und klinischer Versorgung stellen sich relevante und spezifische ethische Fragen im Themenbereich Big Data und personalisierte Medizin. Grund hierfür sind vor allem die unterschiedlichen ethischen Prinzipien, die in der Welt der Forschung und in der ärztlichen Ethik handlungsleitend sind. Während sich Ärzt*innen beispielsweise verpflichtet haben, die Daten ihrer Patient*innen vertraulich zu behandeln und nicht weiterzugeben, wird von Forscher*innen erwartet, ihre Daten im Geiste der „offenen Wissenschaft“ („open science“) weiterzugeben auch damit die Forschungsgemeinschaft die Ergebnisse validiert und der Erkenntnisprozess auf der Grundlage neuer Daten optimiert werden kann (siehe Molnár-Gábor/ Merk, Kap. 16). Ärzt*innen unterliegen zudem den Prinzipien guter klinischer Praxis und dem Stand der ärztlichen Heilkunst, während Forscher*innen ausschließlich den Prinzipien guter wissenschaftlicher Praxis verpflichtet sind. Bisher sind letztere weder für die Therapiewahl für einzelne Patient*innen noch für die Meldung von Ergebnissen aus der Forschungsdatenanalyse verantwortlich. Das Ziel der Forschung ist schließlich, verallgemeinerbares Wissen zu generieren, nicht der unmittelbare individuelle Nutzen für die Gesundheit individueller Patient*innen. International wird derzeit intensiv diskutiert, wie die ethischen und rechtlichen Rahmenbedingungen und die Infrastrukturen in Kliniken und Forschungsinstituten in einer Weise gestaltet werden können, die die Prinzipien dieser beiden Welten in Einklang bringt oder gut begründet gegeneinander abwägt.

Im Folgenden werden wichtige ethische Fragen, die in Deutschland und international diskutiert werden, aufgegriffen: der Umgang mit gesundheitsrelevanten Zusatzinformationen aus dem Forschungskontext, neue Verantwortung für Forscher*innen, Datennutzer*innen und deren Institutionen und die berechtigten moralischen Interessen und Rechte der Patient*innen mit Blick auf die Nutzung ihrer Daten und ihre Möglichkeiten, diese mitzubestimmen. 


\subsubsection{Umgang mit gesundheitsrelevanten Zusatzinformationen aus} dem Forschungskontext

Eine intensive ethische Debatte wurde in den letzten Jahren über den richtigen Umgang mit gesundheitsrelevanten Zusatzinformationen geführt, die im Rahmen des Forschungskontextes auftauchen. Dabei wurde insbesondere auch das Thema „zufälliger“ Befunde analysiert. Ein zufälliger Befund ist „ein Befund in Bezug auf einen einzelnen Forschungsteilnehmer, der potenzielle gesundheitliche oder reproduktive Bedeutung hat und im Verlauf der Forschung entdeckt wird, aber über die Ziele der Studie hinausgeht“ (Wolf et al., 2008). Zufällige Befunde können sich beispielsweise ergeben, wenn Forscher*innen bei der Untersuchung des gesamten Genoms einer Tumorzelle und dem Abgleich mit dem Erbgut gesunder Zellen derselben Person auf genetische Veränderungen stoßen, die auf Risiken für andere Krankheiten als Krebs hinweisen. Die Antizipation von zufälligen Befunden, die im Forschungsumfeld entstehen, wirft eine Reihe ethischer und rechtlicher Fragen auf: hinsichtlich der Gültigkeit und des informativen Werts solcher Befunde, der potenziellen Verantwortung der Forscher*innen, solche Befunde an die behandelnden Ärzt*innen oder an Patient*innen zu kommunizieren und die Patienten*innen im Rahmen des Aufklärungs- und Einwilligungsprozesses darauf vorzubereiten sowie hinsichtlich unterschiedlicher Möglichkeiten, solche Informationen an die jeweiligen Forschungsteilnehmende zurückzumelden, wenn dies von ihnen gewünscht wird (Wolf, 2013; Zawati/Knoppers, 2012).

Während der Umgang mit Zufallsbefunden in der klinischen Welt klar durch die Nachverfolgungs- und Mitteilungspflicht geregelt ist (EURAT, 2015; zu Zufallsbefunden in der Gendiagnostik siehe Mundlos, Kap. 4), gab es für den Forschungsbereich keine derartigen Richtlinien. Dies ist nicht überraschend, da das Ziel der Forschung darin besteht, verallgemeinerbares Wissen zu erlangen und nicht, unmittelbar die Versorgung der Patient*innen zu verbessern. Gegner*innen einer Rückmeldung solcher $\mathrm{Zu}-$ satzinformationen argumentieren mit dieser wichtigen Unterscheidung zwischen Forschung und klinischer Versorgung - nämlich, dass das Handeln von Forscher*innen sich weder am besten Interesse einzelner Patienten*innen zu orientieren habe, noch die Mittel zur Validierung von Befunden zur Verfügung stünden, die in nicht klinisch zertifizierten Forschungslabors gewonnen wurden. Zudem hätten viele Forscher*innen im Gegensatz zu Ärzt*innen nicht das nötige Wissen, das eine Beurteilung der klinischen Relevanz der Ergebnisse ermöglichen würde (Clayton/McGuire, 2012; Ossorio, 2012). Mit dem zunehmenden Einsatz der Genomsequenzierung in patient*innennahen klinischen Studien scheint sich jedoch ein Konsens abzuzeichnen, dass zumindest für direkt handlungsrelevante Ergebnisse die Möglichkeit der Rückmeldung angeboten werden sollte (Bredenoord et al., 2011; EURAT, 2015; Thorogood et al., 2019). Für eine 
Rückmeldepflicht sprechen Fürsorgeüberlegungen, aber auch Reziprozitätsargumente in dem Sinne, dass Forschungsteilnehmenden als Gegenleistung dafür, dass sie ihre Daten für die Wissenschaft zur Verfügung stellen, gesundheitsrelevante Zusatzinformationen nicht vorenthalten werden sollten.

Dabei ist jedoch zu beachten, dass sich zufällige Befunde in der Klinik von genetischen Informationen aus dem Forschungskontext unterscheiden, da letztere eine diagnostische Validierung erfordern, eher auf ein Krankheitsrisiko als auf das Vorhandensein einer Krankheit hinweisen (siehe Mundlos, Kap. 4) und selten sind, da Forscher*innen nicht regelmäßig über genetische Diagnosen stolpern. Daher ist es wichtig, die Umstände zu definieren, die die Rückmeldung von Ergebnissen aus der Forschungswelt zum gesundheitlichen Nutzen einzelner Patient*innen erfordern.

Die Deutsche Gesellschaft für Humangenetik empfiehlt die Rückmeldung von Ergebnissen nur dann, wenn sie analytisch validiert und gesundheitsrelevant sind. Welche Befunde der/die Patient*in erfahren möchte, muss mit ihnen daher schon bei der Aufklärung zur Studienteilnahme besprochen werden. Aufgrund der Vielfältigkeit der Befunde mit sehr unterschiedlicher Relevanz für die jeweiligen Patient*innen und ihre Angehörigen kann eine Vorab-Aufklärung nur beispielhaft anhand bestimmter Krankheitskategorien erfolgen. In der Aufklärung sollte der oder die Patient*in grundsätzlich der Rückmeldung von Zusatzbefunden zustimmen oder widersprechen können.

Auch die interdisziplinäre EURAT-Gruppe zu „ethischen und rechtlichen Aspekten der Totalgenomsequenzierung “, in der Wissenschaftler*innen aus den Lebens-, Sozialund Normwissenschaften an normativ fundierten Empfehlungen für die Handlungspraxis arbeiten, hat zum Thema Rückmeldung von Zusatzbefunden eine Stellungnahme veröffentlicht. Die EURAT-Gruppe schlägt ein abgestuftes Konzept vor. Dieses unterscheidet zwischen der Rückmeldung von therapie- oder vorsorgerelevanten Befunden und solchen, die Wissen enthalten, das zwar keine Therapiemöglichkeiten eröffnet, aber vielleicht für die Lebensplanung wichtig ist. Patient*innen werden gebeten, hierzu ihre Wünsche in einem Statement im Rahmen der Aufklärung zu Forschungsstudien mit Genomsequenzierung zu hinterlegen. Wenn dann im Forschungskontext ein potenziell gesundheitsrelevantes Ergebnis auffällt, soll dieses an die behandlungsführenden Ärzt*innen gemeldet werden, die, je nach Einschätzung der Relevanz und im Abgleich mit dem Patient*innenwunsch, eine Validierung und Rückmeldung veranlassen (EURAT, 2015). 


\subsubsection{Privatsphäre und Schutz vor Diskriminierung - Welche Verantwortung} tragen Forscher*innen und deren Institutionen?

Obwohl Daten für Forschungszwecke ganz überwiegend verschlüsselt weitergegeben werden, können Daten, gerade wenn sie mit anderen Daten abgeglichen werden, doch Rückschlüsse auf die Person, von der sie stammen, zulassen.

Traditionell galten bestimmte Arten von Daten - wie Informationen über Gesundheit, sexuelle Orientierung, ethnische Identität oder Gewerkschaftszugehörigkeit - als besonders gefährlich, wenn sie in die falschen Hände gerieten. Solche Daten werden als sensible Daten bezeichnet, und viele Datenschutzregelungen sehen besondere Schutzmaßnahmen für sie vor. Heutzutage kann nahezu jeder Datensatz - wenn er mit einer ausreichenden Art und Menge anderer Datensätze verknüpft wird - nicht nur zur Identifizierung der Person führen, von der er stammt, sondern auch dazu verwendet werden, Rückschlüsse auf bestimmte Personengruppen zu ziehen. Scheinbar harmlose Informationen über eine Person, wie z. B., ob sie Sportartikel online kauft oder nicht, oder ob sie eine Festnetztelefonnummer hat oder nicht, könnten zu einer Diskriminierung dieser Person führen, wenn sich in anderen Studien oder Datensätzen herausgestellt hat, dass diese Merkmale mit vermeintlich riskantem, ungesundem oder anderweitig kostspieligem Verhalten korrelieren (Dixon/Gellman, 2014).

Genomdaten zählt man zu den besonders sensiblen Daten (siehe Alex/Winkler, Kap. 13). Sie sind ab einer bestimmten Menge spezifisch und damit eine eindeutige Kennung eines Individuums. Zudem können sie Rückschlüsse auf familiäre Beziehungen und Erbkrankheiten zulassen. Informatiker*innen haben gezeigt, dass es mehrere Möglichkeiten gibt, wie ein/e technisch versierte/r Hacker*in die Identität von Teilnehmenden an genetischen Datenbanken aufdecken kann - indem er oder sie Genomdaten beispielsweise mit öffentlich verfügbaren Genealogie-Datenbanken vergleicht (Gymrek et al., 2013; Erlich/Narayanan, 2014). Während mehrere empirische Studien eine positive Einstellung von Personen zur Genomforschung und deren Wert zeigen, waren Bedrohungen der Privatsphäre die wichtigsten Bedenken, auch wenn sie die Bereitschaft zur Teilnahme nicht immer negativ beeinflussen (Pullman et al., 2012; Husedzinovic et al., 2015). In einer Studie über die Ansichten von Krebspatient*innen zu Datenschutzrisiken im Zusammenhang mit der Genomforschung äußerten die Patient*innen im Allgemeinen ihr Vertrauen in Forschungseinrichtungen und einzelne Forscher*innen, äußerten jedoch Erwartungen hinsichtlich institutioneller Schutzmaßnahmen auf der Ebene der Governance - d. h. Führung und Aufsicht (Schleidgen et al., 2019). Sie betreffen technische Schutzmaßnahmen und Governance, Transparenz und Kommunikationsstrategien. Erstens können Re-Identifzierungsrisiken durch technische Maßnahmen wie Zugangskontrollen, differenzierte Datenschutzlösungen 
und kryptografische Techniken verringert werden (Erlich/Narayanan, 2014). Zweitens können ethische Normen und gesetzliche Verbote das Risiko eines Missbrauchs genetischer Daten unabhängig von der theoretischen Möglichkeit zur Re-Identifizierung verringern - z. B. ist im Vereinigten Königreich die wissentliche oder unberechtigte Re-identifizierung einer Person anhand verschlüsselter Daten eine Straftat. Die zuvor erwähnte EURAT-Grundsatzerklärung enthält einen Verhaltenskodex für das wissenschaftliche Personal, das mit Sequenzierungsdaten umgeht, einen Datenschutzrahmen und eine offene Kommunikation über die Risiken für eine Re-Identifizierung in den Aufklärungsdokumenten (EURAT, 2015). In ähnlicher Weise hat eine große Plattform für den weltweiten Datenaustausch zur Genomforschung GA4GH einen Rahmen für einen verantwortungsvollen Datenaustausch mit Leitprinzipien (Knoppers, 2014) und konkreten Instrumenten für die Umsetzung entwickelt. Dies ist die dritte Strategie - nämlich transparent über Risiken der Re-Identifizierung aufzuklären. Die Datenlage, inwieweit es tatsächlich zu einer ungerechtfertigten Benachteiligung von Menschen aufgrund genetischer Informationen kommt, ist noch sehr dünn. In einer systematischen Literaturanalyse von 33 Studien zum Thema genetische Diskriminierung mit einem besonderen Fokus auf Lebensversicherungen kam eine Mehrheit der Studien (58 \%) zu dem Schluss, dass genetische Diskriminierung im Zusammenhang mit Lebensversicherungen ein vernachlässigbares Thema ist, das die bisherige erhebliche gesellschaftliche Debatte und politische Besorgnis nicht rechtfertigt. $42 \%$ kamen hingegen zu dem Schluss, dass genetische Diskriminierung den Zugang zu Lebensversicherungen erschwert. Die Literaturanalyse zeigte jedoch vor allem relevante Lücken in der Datenlage, denn die Mehrheit der Studien konzentrierte sich auf einzelne gut beschriebene genetische Erkrankungen. Forschung zu genetischer Diskriminierung in den Bereichen personalisierte Medizin, Pharmakogenomik oder Infektionskrankheiten fehlt hingegen vollständig. Das macht es besonders schwierig, aus den Ergebnissen des systematischen Literaturvergleichs eine allgemeine, belastbare Schlussfolgerung zur genetischen Diskriminierung zu ziehen, die auf die gesamte Forschung oder den klinisch-genetischen Kontext anwendbar ist (Joly et al., 2013). Diese Lücke zu schließen, wäre wünschenswert, um ausgewogen über Nutzen und Risiken informieren zu können.

\subsubsection{Informierte Zustimmung und Governance: zwei Säulen vertrauenswürdiger Datennutzung}

Entsprechend der ursprünglichen Vision, alle relevanten Informationen für die Steuerung der Behandlung und Prävention von Erkrankungen zu sammeln, beruhen Prä- 
zisionsmedizinprogramme auf der Erfassung, Weitergabe und Integration von Daten aus großen und diversen Datensätzen wie Biomarker- und Omics-Daten sowie der klinischen Verlaufsdokumentation in elektronischen Gesundheitsakten und LebensstilDaten. Das Sammeln von Patient*innendaten für zukünftige Forschungsprojekte mit noch nicht festgelegten Zielen stellt den anerkannten ethischen Standard der spezifischen und informierten Zustimmung in Frage, der genaue Informationen über die Ziele, Methoden, Vorteile und Risiken einer Studie fordert. Die World Medical Association hat auch aus diesem Grund in ihrer Überarbeitung der Deklaration von Helsinki im Jahr 2013 die Bestimmungen zur Forschung mit personenbeziehbaren Daten verändert. Die Deklaration fordert weiterhin eine Einverständniserklärung zur Sammlung, Lagerung und Wiederverwendung von personenbeziehbaren Daten, erwähnt jedoch nicht die Notwendigkeit spezifischer Informationen in Bezug auf künftige Studien (WMA, 2013). Ebenso erkannte CIOMS/WHO eine breite Zustimmung („broad consent“), also eine Zustimmung, die nicht eng auf eine genau bezeichnete Studie, sondern breit für mehrere mögliche Nutzungen aufklärt, als angemessen für solche Studien an (Knoppers/Thorogood, 2017). Eine breite Zustimmung ist zu einer umsetzbaren Lösung geworden, um die Teilnehmer*innen generell über den Umfang der Forschungsprojekte zu informieren, die von ihren Daten gespeist werden, sowie über die Forschungspartner und Länder, die künftig am Datenaustausch beteiligt sind. Eine breite Zustimmung verlagert jedoch die Verantwortung für die Kontrolle und Überwachung der Gültigkeit dieser Zustimmung auf die Forschungseinrichtung, Forschungsethikkommissionen, Datenzugriffskomitees und die Forscher*innen selbst, da sie als Treuhänder*innen für die Interessen der Patienten*innen fungieren müssen. Die Erfüllung dieser Treuhandpflicht kann auf lokaler oder nationaler Ebene funktionieren, wird jedoch für den globalen Datenaustausch ein ehrgeiziges Unterfangen. Daher sind globale Standards für den Datenaustausch und Richtlinien zur Überwachung notwendig, und es gibt Plattformen wie GA4GH, die daran arbeiten.

\subsection{Ethische Begründungen für die und Anforderungen an die Governance der Datennutzung und deren Umsetzung}

Dass digitale Daten in vielen Kontexten leichter erhoben, integriert und analysiert werden können als Daten im Papierzeitalter, bringt vielfältige Chancen für neue und wichtige Erkenntnisse, aber auch neue Risiken für Menschen mit sich - wie beispielsweise das bereits angesprochene Problem der Re-Identifizierbarkeit. Zugleich besteht ein zunehmendes Machtgefälle zwischen den Bürger*innen, von denen die Daten stammen, und denen, die sie nutzen. $\mathrm{Zu}$ den letzteren gehören nicht nur Krankenhäuser und 
öffentliche Forschungsinstitute, sondern zunehmend auch profitorientierte private Unternehmen (Sharon, 2016; Prainsack, 2020). Vor diesem Hintergrund ist gute DatenGovernance wichtiger und dringlicher denn je. Der Deutsche Ethikrat unterstreicht in diesem Zusammenhang die Bedeutung des Konzepts der Datensouveränität, das kein genau bestimmter Rechtsbegriff ist, sondern eine Fülle von Ansätzen und Instrumenten beschreibt, die eine „den Chancen und Risiken von Big Data angemessene, verantwortliche, informationelle Freiheitsgestaltung“" gewährleisten (DER, 2017: 252; Kirschsieper, 2016; Hummel et al., 2021). Formen der Ausgestaltung von Datensouveränität auf der Ebene von Praxis und Technologie sind z. B. dynamische Einwilligungsmodelle („dynamic consent“; Kaye et al., 2015), bei denen Studienteilnehmende für jede Studie erneut angefragt werden oder elektronisch mitbestimmen, ob überhaupt und wann sie gefragt werden (Dove et al., 2012).

Bisher bestand die wichtigste Reaktion auf die Herausforderung des Datenschutzes im Zeitalter der digitalen Forschung und Praxis darin, der und dem Einzelnen mehr Kontrolle darüber zu geben, wie ihre oder seine Daten verwendet werden. In der deutschen Rechtsprechung ist dieses informationelle Selbstbestimmungsrecht schon seit dem sogenannten Volkszählungsurteil von 1983 als Grundrecht anerkannt. Grundlage für das Bundesverfassungsgerichtsurteil damals war das Allgemeine Persönlichkeitsrecht, also Art. 2 Abs. 1 GG in Verbindung mit Art. 1 Abs. 1 GG (unter C II 1. des Urteils; Rn. 152).

Auf europäischer Ebene ist die Datenschutz-Grundverordnung (DSGVO) der Europäischen Union das wichtigste Regelwerk, das Betroffenenrechte in der Datennutzung schützt. Insbesondere klinische Daten werden im Rahmen der DSGVO als besonders sensitiv eingestuft. Sie fordert daher einerseits ein hohes Schutzniveau für sensible Daten, sieht aber im Gegenzug zahlreiche Regelungen zur Privilegierung der Datenverarbeitung zu Forschungszwecken. Voraussetzung für diese privilegierte Nutzung sind geeignete Garantien, die die Betroffenenrechte schützen - wie beispielsweise Zustimmungs- oder Widerspruchslösungen (Art. 9 Abs. 2 lit. j, 89 DSGVO, siehe auch: Spitz et al., 2021). Die DSGVO sieht eine Forschungsprivilegierung insbesondere durch die Öffnungsklausel für nationale Rechtsgrundlagen der Forschungsdatenverarbeitung vor, die von den EU-Mitgliedsstaaten genutzt werden können. Bislang wurde in Deutschland diese Öffnungsklausel durch die Forschungsklausel des § 27 Abs. 1 BDSG (Bundesdatenschutzgesetz) eher restriktiv konkretisiert, da beispielsweise für eine Datenverarbeitung zu Forschungszwecken ohne Einwilligung ein erhebliches Überwiegen der Forschungsinteressen gegenüber den Betroffeneninteressen gefordert wird (§ 22 Abs. 2 S. 2 BDSG). 
Im Folgenden soll jedoch weniger der rechtliche Rahmen, sondern vielmehr die ethischen Überlegungen skizziert werden, die dafür sprechen, dass Bürger*innen ihre klinischen Daten der Forschung zur Verfügung stellen. Zum anderen sollen Möglichkeiten der Mitbestimmung und Teilhabe am Forschungsprozess vorgestellt werden.

\subsubsection{Solidarität als Grundlage und Rahmen für verantwortliche Datennutzung}

Wie eine der Autor*innen dieses Beitrages (BP) an anderer Stelle argumentiert hat, bietet das Konzept der Solidarität einen fruchtbaren Rahmen, um die kollektive Verantwortung für (und Kontrolle über) gute Datennutzung zu stärken (zu Kollektivität und Solidarität als bioethische Konzepte siehe Schicktanz/Buhr, Kap. 18). Solidarität bezieht sich auf die Bereitschaft von Menschen, andere Menschen, mit denen sie in einer im spezifischen Kontext relevanten Hinsicht verbunden sind, zu unterstützen auch wenn es sie etwas „kostet“ (Prainsack/Buyx, 2017). Der Begriff der Kosten ist hier im weitesten Sinne des Wortes zu verstehen; er umfasst finanzielle, praktische oder andere Zuwendungen. Die Verbundenheit, die Menschen mit anderen empfinden und die zur Handlungsgrundlage von Solidarität wird, kann etwas Identitätsstiftendes und Dauerhaftes sein, wie ein gemeinsamer Glaube, der Kampf gegen Unterdrückung, oder die Verfolgung eines politischen Zieles. Sie kann aber auch aus einer spezifischen momentanen Situation heraus entstehen, wie die zeitlich beschränkte Verbundenheit zweier Menschen, die sich gegenseitig über einen kleinen Unfall hinweghelfen. Der entscheidende Punkt ist, dass Solidarität Unterschiede nicht ausblendet oder leugnet; sie findet vielmehr trotz der Unterschiede zwischen Menschen statt, indem das Gemeinsame handlungsleitend wird.

Warum ist ein auf Solidarität basierender Ansatz ein guter Rahmen für Daten-Governance? Dies ist deshalb der Fall, weil er sich auf Situationen konzentriert, in denen Menschen auf der Grundlage dessen handeln, was sie als ihre Gemeinsamkeiten mit anderen betrachten, und nicht auf der Grundlage ihrer Unterschiede. Solidarität im Kontext der Daten-Governance schließt sowohl die gegenseitige Unterstützung der Bürger*innen als „Datensubjekte“ - also als Menschen, deren Daten zu Forschungsund anderen nützlichen Zwecken verwendet werden, - mit ein als auch institutionalisierte Formen von Solidarität: Letztere beinhalten Regeln, Instrumente und Normen, die etwa eine im Sinne der Solidarität gerechte Verteilung von Lasten und Pflichten sicherstellen (siehe z. B. McMahon et al., 2020). Auf diese Weise ist der Solidaritätsansatz gut dazu geeignet, an wesentlichen Stellen die unproduktive Dichotomie zwischen individuellen Interessen und dem kollektiven Wohl aufzubrechen. Ein solidaritätsbasierter Ansatz hebt Praktiken, Instrumente und Strukturen hervor, in denen Menschen 
ihre eigenen Bedürfnisse und Interessen auch als die anderer Menschen erkennen (und umgekehrt) und er unterstützt die Verfestigung solcher Praktiken in institutionellen und rechtlichen Arrangements.

Formen der Datennutzung, die aus Sicht des öffentlichen Interesses wünschenswert sind, sollten aus dieser Perspektive unterstützt werden - gegebenenfalls durch Erleichterung der regulatorischen Anforderungen für solche Datennutzungen oder durch Bereitstellung öffentlicher Mittel (Prainsack, 2017b). Dies würde auch dem Problem begegnen, dass es heute für viele im öffentlichen Interesse handelnde Institutionen insbesondere innerhalb des Gesundheitswesens - viel strengere ethische und regulatorische Auflagen gibt als für private Konzerne, die mit den von ihren Nutzer*innen gesammelten Daten viel freier verfahren können, weil die Datennutzer*innen mit der Nutzung der Plattform, der App oder des Onlinedienstes formal der Verwendung der Daten zugestimmt haben.

Für im öffentlichen Interesse stehende Formen der Datennutzung sollte es einfacher werden, auf Datensätze zuzugreifen und sie zu nutzen. Derzeit können einige wertvolle Forschungsarbeiten - im Kontext der personalisierten Medizin und in anderen Bereichen - aufgrund der eher restriktiven Auslegung der an und für sich forschungsfreundlichen DSGVO durch das Bundesdatenschutzgesetz und die föderalen, zeitintensiven forschungsethischen Prüfungsverfahren nicht realisiert werden. Ein solidarischer Ansatz für Data Governance schlägt vor, dass diese Barrieren für Datennutzungen, die die Kriterien für „wünschenswerte“ Datennutzungen erfüllen, deutlich reduziert werden sollten. Wie bereits erwähnt, kann der Abbau von Barrieren die Erleichterung von Zustimmungserfordernissen oder die Beschleunigung und Entlastung von forschungsethischen Prüfungen beinhalten.

Gleichzeitig erkennt ein solidaritätsbasierter Daten-Governance-Ansatz an, dass selbst wünschenswerte Datennutzungen regelmäßig zumindest geringe Risiken für eine kleine Anzahl von Personen mit sich bringen. Ein auf Solidarität basierender Rahmen kann dazu beitragen, dass Strategien zur Schadensbegrenzung vorhanden sind, falls tatsächlich ein Schaden eintritt. Daher ist ein weiteres wichtiges Element innerhalb dieses Ansatzes die Schadensminderung. Sogenannte Schadensminderungsorganisationen („harm mitigation bodies“; siehe McMahon et al., 2020) etwa würden auch Menschen offenstehen, die keinen Zugang zu Rechtsmitteln haben, weil sie z. B. keinen Nachweis darüber führen können, dass eine bestimmte Handlung oder Unterlassung seitens einer datenverarbeitenden Rechtsperson den konkreten Schaden verursacht hat. Eine Schadensminderungsorganisation hätte drei Hauptfunktionen: Erstens wäre sie ein Ort, an dem Informationen über die Arten von Schaden, die Menschen empfinden, systematisch zusammenlaufen. Wenn bestimmte Probleme gehäuft auftreten, 
könnten die Praktiken seitens der Datennutzer*innen entsprechend verbessert werden. Zweitens wäre die Schadensminderungsorganisation eine Stelle, die die betroffenen Menschen „sieht“ und wahrnimmt und ihnen auch darlegen kann, was schief gelaufen ist (oder nicht) - es wäre dahingehend ein wichtiger Schritt in Richtung von mehr Transparenz und Interaktion auf Augenhöhe. Drittens könnten Schadenminderungsorganisationen, die idealerweise durch Beiträge datennutzender Organisationen ab einer bestimmten Größe finanziert würden, in Härtefällen auch Menschen, die finanziellen Schaden erlitten haben, durch Geldzahlungen unterstützen (diese Geldzahlungen wären kein Schadenersatz im rechtlichen Sinne, sondern explizit eine Hilfestellung für Härtefälle, die nicht unbedingt der genauen Höhe des entstandenen finanziellen Schadens entsprechen muss; siehe McMahon et al., 2020).

\subsubsection{Hilfs- und Gemeinwohlpflichten als Begründung eines normativen Anspruchs an die Bürger, ihre klinischen Daten der Forschung zur Verfügung zu stellen}

Da das Solidaritätsprinzip einen Rahmen für die sekundäre Nutzung von Daten beschreibt, aber aus ihm keine Pflicht zur Teilnahme an der Forschung abgeleitet werden kann, gilt es mit Blick auf das Thema Big Data und personalisierte Medizin zu klären, ob und welche moralischen Pflichten Bürger*innen haben, ihre klinischen Daten für die Forschung zur Verfügung zu stellen. ${ }^{2}$ Jungkunz et al. führen eine solche potenzielle moralische Pflicht auf eine allgemeine Hilfspflicht und Gemeinwohlverpflichtungen zurück - vorausgesetzt, bestimmte Bedingungen sind erfüllt (Jungkunz et al., im Druck).

In ihrer Argumentation beziehen sich Jungkunz et al. auf die liberale Tradition nach Kant und Rawls. Die allgemeine Hilfspflicht beschreibt Kant als „Pflicht der Wohltätigkeit“ (Kant, 1797/2017). Kant begründet diese Pflicht ex negativo damit, dass das Unterlassen einer Hilfeleistung im Angesicht der Hilfsbedürftigkeit anderer niemals als allgemeines Gesetz gewollt werden kann. ${ }^{3}$ Rawls fügt der kantischen Begründung hinzu, dass die allgemeine Hilfspflicht deshalb als Pflicht in einer liberalen Gesellschaft gelten sollte, weil ihre Etablierung einen entscheidend positiven Einfluss auf die Lebensquali-

2 Zu Solidarität und Partizipation an datenintensiver Medizinforschung siehe auch Schicktanz/ Buhr (Kap. 18).

3 „Denn ein Wille, der dieses beschlösse, würde sich selbst widerstreiten, indem der Fälle sich doch manche ereignen können, wo er anderer Liebe und Teilnehmung bedarf, und wo er durch ein solches aus seinem eigenen Willen entsprungenes Naturgesetz sich selbst alle Hoffnung des Beistandes, den er sich wünscht, rauben würde“ (Kant, AA IV, Grundlegung zur Metaphysik der Sitten: 423). 
tät aller Mitglieder hätte (Rawls, 1979: 374). Die allgemeine Hilfspflicht stellt eine Pflicht dar, anderen in Notsituationen Hilfe zu leisten. Unter Notsituationen ist jedoch nicht ausschließlich ein Szenario zu verstehen, wie es im Kontext von Diskussionen über Hilfeleistung und deren Unterlassung häufig genannt wird, indem z. B. ein Passant ein ertrinkendes Kind aus einem flachen Gewässer rettet. ${ }^{4}$ Vielmehr besteht immer dann eine allgemeine Hilfspflicht, wenn folgende Bedingungen erfüllt sind: (1) Es liegt eine Notsituation bzw. eine ernste Situation der Bedürftigkeit vor, in der eine Person essenzielle Hilfe benötigt, z. B. um Grundgüter zu erlangen, die sie für die Führung eines selbstbestimmten Lebens benötigt. (2) Die von einer Person moralisch eingeforderte Handlung leistet mit hoher Wahrscheinlichkeit effektiv Hilfe. (3) Die Hilfe kann nicht von jeder beliebigen anderen Person durchgeführt werden. (4) Die Hilfeleistung ist zumutbar in Bezug auf das Verhältnis von Kosten für die Hilfe leistende Person und Nutzen für die Hilfe empfangende Person.

Wenn es darum geht, dass Personen ihre klinischen Daten für biomedizinische Forschung bereitstellen, so liegen die genannten vier Bedingungen vor, die eine Hilfspflicht begründen: (1) Kranke Menschen sind aufgrund ihrer Krankheit in einer Notsituation, in der sie Hilfe benötigen. (2) Die Bereitstellung klinischer Daten für biomedizinische Forschung ist mit hoher Wahrscheinlichkeit ein Akt, der zukünftigen Patient*innen hilft. Dadurch, dass Daten beliebig oft über einen beliebig langen Zeitraum in sehr unterschiedlicher Weise genutzt werden können, ist die Wahrscheinlichkeit hoch, dass sie früher oder später zur Erzeugung von Wissen beitragen, welches die Behandlung zukünftiger Patient*innen verbessern kann. (3) Jeder Datensatz ist einzigartig und kann nicht durch beliebige andere Datensätze ersetzt werden. (4) Die Hilfeleistung in Form einer Bereitstellung klinischer Daten für biomedizinische Forschung ist zumutbar insofern ein potenziell großer Nutzen für zukünftige Patient*innen geringen Kosten auf Seiten der Patient*innen gegenübersteht, deren klinische Daten genutzt werden. Voraussetzung für diese geringen Kosten ist jedoch, dass für die Sicherheit der Daten vor etwaigem Missbrauch gesorgt ist (zu den Bedingungen von Sicherheit und Risiken siehe auch Jungkunz et al., 2021).

Die Pflicht zu gemeinwohlförderlichem Handeln ist mit der allgemeinen Hilfspflicht verwandt, spricht aber nicht die individuelle Dimension, sondern komplexe gesellschaftliche Zusammenhänge an. Hierbei spielen Institutionen eine wichtige Rolle, die das Gemeinwohl fördern. Die moralische Pflicht zu gemeinwohlförderlichem Handeln stellt eine Weiterentwicklung der natürlichen „Gerechtigkeitspflicht“ Rawls` dar: die Pflicht, gerechte Institutionen zu fördern und zu unterstützen, wenn dies mit geringem

Vgl. hierzu Singer (1972). 
Aufwand möglich ist. Diese Institutionen sind notwendig für die Stabilität einer gerechten Gesellschaft und somit unterstützenswert (Rawls, 1979: 368 ff.). Gemeinwohlförderliches Handeln geschieht $u$. a. durch die Unterstützung von Institutionen, die die Rechte von Individuen sichern und die gerechte Verteilung sozialer Grundgüter regeln, d. h. Güter, die von allen Menschen unabhängig von individuellen Vorstellungen eines guten Lebens benötigt werden (Bohlken, 2011). Das Gesundheitswesen ist eine Institution, welche diese Grundgüter erzeugt und - im Falle des öffentlichen Gesundheitswesens in Deutschland - für alle zugänglich macht.

Für die Pflicht zu gemeinwohlförderlichem Handeln gelten ähnliche Bedingungen wie für die allgemeine Hilfspflicht. Im Falle der Bereitstellung klinischer Daten für die biomedizinische Forschung, welche dem Gesundheitssystem bei der Erzeugung und Bereitstellung von Grundgütern hilft, indem sie Wissen zur Verbesserung der Behandlung zukünftiger Patient*innen erzeugt, sind diese Bedingungen erfüllt. (1) Krankheit ist in der Gesellschaft stets präsent und ein Bedarf zur Verbesserung der Behandlung ist für viele Krankheiten stets vorhanden. (2) Die biomedizinische Forschung, in der die klinischen Daten genutzt werden, dient dem Gemeinwohl. Biomedizinische Forschung fördert (indirekt) das Grundgut „Gesundheit“, indem sie relevantes Wissen erzeugt, welches der Verbesserung der Behandlung dient. (3) Wie bereits bei der allgemeinen Hilfspflicht erwähnt, ist jeder Datensatz einzigartig und nicht durch jeden anderen ersetzbar; (4) die Datennutzung ist unter gewissen Voraussetzungen zumutbar (siehe oben).

Sowohl die allgemeine Hilfspflicht als auch die Pflicht zu gemeinwohlförderlichem Handeln zeichnen sich durch ihre Universalisierbarkeit aus. So gilt die allgemeine Hilfspflicht allgemein, d. h. gegenüber jedem Anderen, ja sogar gegenüber Personen, die noch nicht geboren sind. Die Unterstützung der biomedizinischen Forschung durch die Bereitstellung der eigenen klinischen Daten ist eine Hilfeleistung gegenüber zukünftigen Generationen von Patient*innen. Die Pflicht zu gemeinwohlförderlichem Handeln zielt zwar aus rein pragmatischen Gründen in erster Linie auf das Gemeinwohl der eigenen Gesellschaft, ist jedoch nicht auf dieses beschränkt. Die Bereitstellung klinischer Daten für die biomedizinische Forschung stellt eine gemeinwohlförderliche Handlung dar, die einen universellen, über die eigene Gesellschaft hinausgehenden Nutzen erzeugt. Die biomedizinische Forschung mit klinischen Daten ist prinzipiell in der Lage, Wissen zu erzeugen, welches in jedem Gesundheitssystem weltweit angewendet werden kann. Dieses Wissen dient neben Patient*innen auch gesunden Personen, da auch diese von einer gesunden Gesellschaft und der Sicherheit, im Krankheitsfall eine gute Behandlung erhalten zu können, profitieren. 


\subsubsection{Formen der Bereitstellung von klinischen Daten für die Forschung - das Beispiel Datenspende}

Für die konkrete Umsetzung einer moralischen Pflicht zur Unterstützung der Forschung mit klinischen Daten wurden bereits verschiedene Wege vorgeschlagen (zum Datenschutzrecht im Kontext der Forschung an Hirnorganoiden siehe Taupitz, Kap. 15, und zum Datenschutz von Neurodaten Molnár-Gábor/Merk, Kap. 16). Vorrang hat sicher weiterhin die informierte Einwilligung der Patient*innen. Diesen Weg geht in Deutschland die Medizininformatik-Initiative. Ihr Ziel ist es, dass alle Patient*innen, die sich in Deutschland in eine Klinik oder Praxis begeben, gefragt werden, ob sie ihre Gesundheitsdaten für die Forschung zur Verfügung stellen. Willigen sie ein, werden die Daten in sogenannten Datenintegrationszentren vor Ort gesammelt. Die Datenintegrationszentren sind so vernetzt, dass sie für Forschungsfragen von Forscher*innen deutschlandweit danach durchsucht werden können, wie viele geeignete Datensätze es hierfür gibt. Nach einem Genehmigungsverfahren und unter Einbeziehung der Ethikkommissionen können die Forschungsprojekte dann auf die Datensätze zugreifen. ${ }^{5}$

Auch in der elektronischen Patientenakte (ePA) sollen Nutzer*innen die Möglichkeit haben, ihre Daten freiwillig zu teilen. Auch hier ist vom Gesetzgeber eine Einwilligungslösung vorgesehen („Opt-In“). Das heißt, die/der Patient*in entscheiden sich aktiv für eine Bereitstellung der Daten, und wer nicht explizit einwilligt, ist nicht eingeschlossen. Eine elektronische Patientenakte können seit dem 1. Januar 2021 alle gesetzlich Versicherten von ihrer Krankenkassen erhalten. In der Akte können medizinische Befunde und Informationen aus vorhergehenden Untersuchungen und Behandlungen über Praxis- und Krankenhausgrenzen hinweg umfassend gespeichert werden (Patientendatenschutzgesetz vom 14.10.2020). ${ }^{6}$

Ein dritter Vorschlag zur Sekundärnutzung von Patient*innenendaten ist die sogenannte Datenspende. Sie entkoppelt zum einen den Zeitpunkt der Einwilligung vom Arztbesuch. Dies ist der Tatsache geschuldet, dass gerade beim Arzt- oder Krankenhausaufenthalt wenig zeitliche und gedankliche Ressourcen vorhanden sind, sich mit dem Thema Sekundärnutzung von Gesundheitsdaten zu beschäftigen. Zudem sind manche Patient*innen so krank, dass sie gar keine Einwilligung unterschreiben können. Dies führt zu einer Verzerrung der Daten und zu einer schlechteren Repräsentation gerade jener Patient*innengruppen, die besonders krank sind und deren Krankheitsbilder möglicherweise besonders der weiteren Erforschung bedürfen. Ähnlich wie bei der Organspende ist also jede Person aufgerufen, sich Gedanken über eine Datenspende zu

5 Siehe unter: https://www.medizininformatik-initiative.de/de/start [01.03.2021].

6 Gesetz zum Schutz elektronischer Patientendaten in der Telematikinfrastruktur (Patientendaten-Schutz-Gesetz - PDSG) vom 14. Oktober 2020. 
machen - ob gesund oder krank. Im Gegensatz zur Organspende basiert das aktuell vorgeschlagene Modell jedoch auf einer Widerspruchslösung. Das heißt, alle Gesundheitsdaten aller Bürger*innen könnten genutzt werden, solange sie der Nutzung nicht widersprechen (BMG, 2020).

Konzeptionell ist der Begriff der Datenspende allerdings problematisch. Während er im Englischen häufig ganz spezifisch auf die Überlassung von Datennutzungsrechten nach dem Tod eines Menschen (eines sogenannten „Datensubjektes“) beschränkt wird (Krutzinna/Floridi, 2019), wird er in der deutschsprachigen Debatte aktuell für eine Praxis verwendet, in der Personen ihre Daten ganz allgemein der Forschung zur Verfügung stellen oder auch Forschungseinrichtungen oder anderen Organisationen überlassen (z. B. DER, 2017; siehe auch Hummel et al., 2019). Diese Begriffswahl birgt einige ontologische und auch praktische Herausforderungen, die offenkundig werden, wenn wir uns die Eigenschaften des Instruments der Spende genauer ansehen. Dabei überträgt jeweils die oder der Eigentümer*in einer Sache diese an eine andere Person oder Einrichtung, ohne eine Gegenleistung dafür zu verlangen. Der letzte Aspekt - dass etwas gegeben wird, ohne eine Gegenleistung zu verlangen - sagt uns zwei Dinge über Spenden: Erstens, dass sie sich außerhalb des Bereichs kommerzialisierter Transaktionen befinden, was bedeutet, dass mit ihrer Übertragung kein wirtschaftliches Gewinnmotiv verbunden ist; und zweitens, dass Spenden nicht direkt reziprok sind. Einige Definitionen von Spenden schlagen das Wort „Geschenk“ als Synonym für Spende vor, um zu betonen, dass Geschenke keineswegs nur in eine Richtung wirken: Sowohl die schenkende als auch die empfangende Person sind in Netzwerke gegenseitiger moralischer und sozialer Verpflichtungen eingebettet, die komplexen Regelwerken unterliegen. Das Verhalten sowohl der schenkenden als auch der beschenkten Person wird durch Erwartungen darüber reguliert, wie und was zu geben und zu empfangen ist, und es drohen in manchen Kontexten soziale Konsequenzen, wenn sich Schenkende und Beschenkte nicht an diese ungeschriebenen Regeln halten (z. B. Caplow, 1984; Carrier, 1991; Bergquist/Ljungberg, 2001; Zeitlyn, 2003; siehe auch Hummel et al., 2019). Mit anderen Worten: Geschenke beruhen häufig indirekt auf Gegenseitigkeit - im Gegensatz zur grundsätzlich einseitigen Datenspende.

Warum sind diese Aspekte wichtig, wenn man über Datenspenden nachdenkt? Zunächst einmal unterstreichen sie den relationalen Charakter von Spenden. Ihr Wert, ihre Folgen und die Praktiken des Gebens und Empfangens von Spenden können nicht außerhalb der persönlichen, sozialen und ökonomischen Beziehungen, in die sie eingebettet sind, verstanden oder beurteilt werden. Damit scheint der Begriff der Spende als nur sehr schlecht auf eine Situation anwendbar, in der eine Person ihre Daten einer Institution (z. B. einem Universitätskrankenhaus, einem außeruniversitären Forschungs- 
institut etc.) oder der Forschung im Allgemeinen zur Verfügung stellt, zu der sie keine persönliche Beziehung hat und von der sie auch keine Gegenleistung erwartet. Zudem kann man argumentieren, dass eine Spende immer etwas betrifft, das „verbraucht“ werden kann, bzw. etwas, das nur begrenzt verfügbar ist: Wenn wir einer Kunstschule Geld spenden, dann können wir dasselbe Geld nicht der Katastrophenhilfe spenden. Wenn wir einer Person eine Niere spenden, können wir dieselbe Niere nicht einer anderen Person spenden. Traditionell impliziert Spende, dass es eine konsumierbare Sache gibt, die von einer Entität auf eine andere übertragen wird - ohne dass sich die spendende Partei Nutzungsrechte vorbehält. Letzteres wäre hinsichtlich von Gesundheitsdaten hoch problematisch; es wäre wohl unethisch, eine Person von der Nutzung ihrer eigenen Gesundheitsdaten auszuschließen. Auch Ersteres - die Konsumierbarkeit - trifft auf Daten nicht in derselben Weise wie auf Geld oder Organe zu, weil sie vervielfältigbar sind und damit kein endliches Gut darstellen.

Im Fall der postmortalen Nutzung von Daten ist der Begriff „Spende“ deshalb noch akzeptabel, weil zumindest die gemeinsame Nutzung der Daten keine Option mehr darstellt. Weil die/der Spender*in ja verstorben ist, können die Nutzungsrechte, die er oder sie vormals innehatte, allesamt auf eine andere Partei übergehen. Über den Kontext der postmortalen Spende hinaus ist der Begriff der Datenspende jedoch nur dort sinnvoll, wo eine nicht-kommerzielle Übertragung von Daten von einer lebenden Person auf eine andere stattfindet, die so weitgehend ist, dass der oder die Spender*in alle Nutzungs- und Verfügungsrechte irreversibel überträgt (ohne sich eigene Nutzungsund Verfügungsrechte vorzubehalten; siehe Prainsack, 2019). Im Kontext der Gesundheitsdaten wäre das aus genannten Gründen ethisch nicht vertretbar.

Ein besserer Begriff für die Zurverfügungstellung von Daten an Dritte zur nicht ausschließlichen Nutzung zu Lebzeiten der Patient*innen ist der Begriff der Datenfreigabe. Dieser Begriff beschreibt, dass Daten - hier vom Datensubjekt - zu bestimmten Nutzungsformen freigegeben werden; wobei der Begriff nichts darüber aussagt, wie eng oder weitgehend diese Nutzungsrechte sind, und auch nicht, welche Formen der Nutzung sich die/der Patient*in selbst vorbehält - oder ob die Freigabe zurückgenommen werden kann. 


\subsection{Partizipation und Empowerment - die Rolle der Patient*innen in datenintensiver und personalisierter Medizin}

Eine Kurzform und Schlagwort für die personalisierte oder Präzisionsmedizin, das vor allem zu Werbezwecken häufig verwendet wird, ist die sogenannte P4 Medizin. Die vier „P‘s“ stehen für prädiktiv, präventiv, personalisiert und partizipativ. Während die ersten drei Versprechen vonseiten der Medizin zunehmend eingelöst werden, ist das vierte besonders offen in seiner Interpretation und unterbestimmt in seiner Bedeutung - es wird häufig bei P4 Medizin mit genannt und beinah nie in den entsprechenden Forschungsprojekten und Protokollen mit Konzepten oder einer konkreten Praxis hinterlegt (Schleidgen et al., 2017; zu Partizipation als bioethisches Konzept siehe auch Schicktanz/Buhr, Kap. 18).

17.5.1 Der inflationäre Gebrauch von „Empowerment“ im Zusammenhang mit personalisierter Medizin

Einige Autor*innen und Praktiker*innen feiern die personalisierte und Präzisionsmedizin in Bausch und Bogen als Form von „Empowerment“ - gerade weil die Mitwirkung der Patient*innen an der Datenerhebung eine Vorbedingung der personalisierten Medizin ist (Prainsack, 2017a). Andere werfen partizipativen Praktiken und Initiativen im Bereich der Medizin vor, die behauptete „Ermächtigung“ der Patient*innen übermäßig zu romantisieren. Wie der Kommunikationswissenschaftler Nathaniel Tkacz (2015: 43) argumentiert, „, bietet die bloße Tatsache der Partizipation [...] keinen substanziellen Zugang zum Verständnis von Transformationen in der tatsächlichen Zusammenarbeit - der qualitativen oder Mikro-Dimension“. Eine Initiative als partizipativ zu etikettieren, sagt nichts darüber aus, wie sich die Verteilung von Macht und Handlungsfähigkeit zwischen den Akteur*innen verändert. Gilles Frydman (2010) beispielsweise definiert „partizipative Medizin“ als „eine Bewegung, in der vernetzte Patient*innen von bloßen Passagier*innen zu verantwortlichen Treiber*innen ihrer Gesundheit werden und in der Anbieter*innen sie als vollwertige Partner*innen ermutigen und wertschätzen“ (siehe auch Hughes et al., 2008). In dieser Sichtweise liegt die primäre Handlungsfähigkeit ironischerweise immer noch bei den Gesundheitsdienstleister*innen, die die Patient*innen als „vollwertige Partner*innen ermutigen und wertschätzen“ sollen; das bedeutet, dass die Patient*innen, um vollständig „ermächtigt“ zu werden, zuerst von anderen ermutigt und wertgeschätzt werden müssen. Um bei dem von Frydman verwendeten Vergleich mit dem Auto zu bleiben: Zwar sind die Lenker*innen diejenigen, 
die das Auto bedienen, aber sie sind nicht immer diejenigen, die entscheiden, wohin die Reise geht. Außerdem können sie das Auto nur bedienen, wenn sie genug Geld haben, um sich überhaupt ein Auto anzuschaffen und die Kosten für Kraftstoff und Wartung zu bezahlen (siehe Prainsack, 2017a).

Mit Blick auf die Gesundheitsversorgung wäre eine „echte“ partizipative Medizin eine, in der die Handlungsoptionen aller Menschen, und insbesondere auch der ökonomisch und sozial Benachteiligten, erweitert würden. Dies bedeutet, dass ein Gesundheitssystem, das alle Menschen unabhängig von ihren individuellen ökonomischen und sozialen Ressourcen gut und nach ihren Bedürfnissen versorgt, eine Vorbedingung sinnvoller Partizipation darstellt. Und auch die weiteren sozialen, ökonomischen und politischen Bedingungen in einer Gesellschaft tragen dazu bei, sinnvolle Partizipation im Gesundheitswesen zu ermöglichen. Ein undifferenziertes Abfeiern jeder Form von Partizipation im Gesundheitssystem unter dem Begriff des „Empowerment“ übertüncht im besten Fall die strukturellen Unterschiede, die determinieren, wann und wo jemand überhaupt teilhaben kann. Im schlechtesten Fall verschleiert eine solch undifferenzierte Sichtweise auf Empowerment die Tatsache, dass mehr Möglichkeiten zur Selbstbestimmung normalerweise jene Menschen noch mehr befähigt, die bereits privilegiert sind. Nur wenn das Fundament - also der Zugang zum Gesundheitssystem - stabil und gerecht ist, kann sichergestellt werden, dass alle Menschen ähnliche Chancen zu sinnvoller Partizipation an medizinischen Entscheidungen ihre eigene Gesundheit betreffend haben - und auch an medizinischer Forschung. Wer bereits in der Gesundheitsversorgung marginalisiert wird, - wie etwa die Gesundheitsbedürfnisse geflüchteter Menschen, die im Gesundheitssystem manchmal nicht „gesehen“ werden (Spahl/Prainsack, 2021) - ist in der datenintensiven Medizin häufig völlig unsichtbar.

\subsubsection{Patient*innen als Forschungsförder*innen und Partner*innen in der personalisierten Medizin}

Neben dem berechtigten Vorbehalt gegenüber der Beteiligung von Patient*innen allein um der Beteiligung willen oder als Lippenbekenntnis, gibt es eine wichtige Entwicklung, wie durch das eigenständige Engagement von Patient*innen die Forschung und der medizinische Erkenntnisgewinn tatsächlich vorangebracht werden (FAZ, 2018). Hierzu gehören zum einen Phänomene wie Gesundheitsplattformen im Internet, in denen sich beispielsweise Patient*innen mit seltenen Erkrankungen (zur Bedeutung der Gendiagnostik für seltene Erkrankungen siehe Mundlos, Kap. 4) vernetzen, austauschen und gegenseitig beraten - wie beispielsweise über 600.000 Patient*innen zu 
2.800 verschiedenen Erkrankungen auf der Plattform „Patients Like Me“. ${ }^{7}$ Dabei fallen große Mengen strukturierter und unstrukturierter Daten an - beispielsweise zu Krankheitsverläufen unter verschiedenen Therapien, deren Verträglichkeit, Wechselund Nebenwirkungen. Die Plattform finanziert sich durch den Verkauf dieser Daten an Hersteller*innen von Medizinprodukten oder die pharmazeutische Industrie, unterstützt aber auch Forschungsprojekte in Kooperation mit universitären Forscher*innen. In den mehr als 100 wissenschaftlichen Publikationen, die laut eigenem Register in Kooperation mit der Plattform zustande kamen, finden sich Messinstrumente zur Lebensqualität und validierte Fragebögen zu patient*innenbezogenen Endpunkten. Die systematische Einbeziehung der Patient*innensicht und -erfahrung ist nicht nur für die Messung patient*innenbezogener Endpunkte relevant, sondern kann dabei helfen, Versorgungslücken oder intersektorale Engpässe zu identifizieren. Sie ist auch für Informations- und Aufklärungsmaterialien gerade in der Forschung zur Präzisionsmedizin essenziell. Am Nationalen Centrum für Tumorerkrankungen in Heidelberg wurde beispielsweise die Patient*inneninformation zu komplexen Themen der Genomsequenzierung in Inhalt, Gewichtung und Darstellung in Diskussionsrunden gemeinsam mit Patient*innen entwickelt (Husedzinovic et al., 2015). Ein/e Vertreter*in des Patient*innenbeirats ist dabei, wenn neue Forschungsstudien vorgestellt und diskutiert werden und benennt die Patient*innen-Expert*innen, die die Studieninformationen auf Verständlichkeit prüfen.

Patient*innen als Expert*innen und Forschungspartner*innen - das ist eine neue Rolle, die besonders in der Forschung zu seltenen Erkrankungen, aber auch in der personalisierten Krebsmedizin, in den letzten 20 Jahren an Bedeutung gewinnt (Supple et al., 2015). Vor allem im Vereinigten Königreich, aber auch in den EU-finanzierten Forschungsprojekten, ist die Beteiligung von Patient*innen ein wichtiges Kriterium für die Forschungsförderung. Die Idee dabei ist, dass Patient*innen von Anfang eines Forschungs- und Entwicklungsprozesses an als Forschungspartner*innen eingebunden sind. Sie wählen das Forschungsthema aus, bringen Themen mit ein, die für die spezielle Erkrankung relevant sind, diskutieren mit bei der Entwicklung des Forschungsdesigns, sind Mitantragsteller*innen bei der Forschungsförderung, helfen bei der Rekrutierung von Studienteilnehmer*innen, der Auswertung und Verbreitung der Studienergebnisse. Hierfür müssen sie tatsächliche Expert*innen für die jeweilige Erkrankung sein nicht nur mit Blick auf die persönliche Erkrankungsgeschichte, sondern die naturwissenschaftlichen Zusammenhänge. Ein Beispiel dafür, wie erfolgreich Patient*innen die Forschung voranbringen können, ist Sharon Terry. Sie hat 1994, als ihre beiden Töchter 
mit einer sehr seltenen genetischen Erkrankung - Pseudoxanthoma Elasticum - diagnostiziert wurden, eine gemeinnützige Organisation zur Erforschung der Erkrankung gegründet. Sie warb das Geld für die Forschung ein, rekrutierte über eine selbst gegründete Internetplattform mit Register und eigener Biobank betroffene Familien, ${ }^{8}$ stellte ein Forschungsteam zusammen und patentierte einen genetischen Test für die Erkrankung.

In diesem Ansatz geht es weniger um die Beteiligung der Beteiligung wegen, sondern um die Verbesserung und Patient*innenorientierung des Forschungsprozesses und dessen Ergebnisse (Geissler et al., 2017). Damit Patient*innen ein solch vertieftes Wissen erlangen können, werden auf europäischer wie nationaler Ebene zunehmend Patient*innenakademien eingerichtet. Auch in Deutschland hat das BMBF für die neu ausgelobte Förderung und Neugründung Nationaler Zentren für Tumorerkrankungen die Beteiligung von Patient*innen zu einem wichtigen Bewertungskriterium erhoben. Dies bietet auch für Deutschland die Chance, Patient*innenbeteiligung so zu gestalten, dass sie sich positiv auf Forschungsprozesse und -ergebnisse auswirkt.

\subsection{Fazit}

Dieser Beitrag hat sich mit ethischen Fragen an der Schnittstelle von datenintensiver (Big Data) Medizin und Personalisierung und insbesondere auch mit der Rolle und Partizipation von Patient*innen in diesem Kontext beschäftigt. Ansätze, die Big Data nicht nur als rein technisches Phänomen, sondern als eine soziotechnische Praxis sehen, haben hier den Vorteil, dass sie die gesellschaftlichen, ökonomischen und politischen Aspekte mit im Blick haben, die mitbestimmen, wie die neuen Praktiken medizinischer Forschung oder neue Technologien in der Diagnose und Therapie genutzt werden. Für eine datenintensive, personalisierte Medizin, die bestehende Ungleichheiten im Gesundheitswesen nicht vergrößert und allen Patient*innengruppen gleichermaßen zugutekommt, ist es zudem notwendig, Ungleichheiten auch in anderen Lebens- und Praxisbereichen, beispielsweise mit Blick auf die Gesundheitskompetenz zu reduzieren. Dasselbe gilt für Daten: Nachdem heute in einer Zeit, in der aufgrund der Verknüpfbarkeit unterschiedlicher Datensätze aus fast allen Arten von Daten und Informationen gesundheitsrelevante Schlüsse gezogen werden können, umfasst die ethische Governance der „Gesundheitsdaten“ fast alle Lebensbereiche. Auch hier sprechen wir uns für eine gute Balance der Stärkung individueller Kontrolle über die Nutzung der eigenen Daten einerseits und der Stärkung der Nutzung der Daten zum Wohle aller aus.

8 Siehe unter: http://www.geneticalliance.org/ [03.03.2021]. 


\subsection{Literaturverzeichnis}

Ausiello, D./Lipnick, S. (2014): Quantitative human phenotyping: The next frontier in medicine. In: Transactions of the American Clinical and Climatological Association 125: 219-228.

Ausiello, D./Lipnick, S. (2015): Real-time assessment of wellness and disease in daily life. In: Big data 3(3): 203-208.

BEK =[Österreichische] Bioethikkommision (2019): Ärztliches Handeln im Spannungsfeld von Big Data, Künstlicher Intelligenz und menschlicher Erfahrung. Unter: https://www.bundeskanzleramt. gv.at/themen/bioethikkommission/publikationen-bioethik.html [25.02.2021].

Bergquist, M./Ljungberg, J. (2001): The power of gifts: organizing social relationships in open source communities. In: Information Systems Journal 11(4): 305-320.

BMG = Bundesministerium für Gesundheit (2020): Wissenschaftliches Gutachten: „Datenspende“

- Bedarf für die Forschung, ethische Bewertung, rechtliche, informationstechnologische und organisatorische Rahmenbedingungen. Unter: https://www.bundesgesundheitsministerium. de/fileadmin/Dateien/5_Publikationen/Ministerium/Berichte/Gutachten_Datenspende.pdf [01.03.2021].

Bohlken, E. (2011): Die Verantwortung der Eliten. Campus Verlag, Frankfurt am Main.

Bredenoord, A. L. et al. (2011): Disclosure of individual genetic data to research participants: the debate reconsidered. In: Trends Genet 27(2): 41-47.

Bruynseels, K. et al. (2018): Digital twins in health care: ethical implications of an emerging engineering paradigm. In: Frontiers in genetics 9: 31, Online-Publikation 13.02.2018. DOI: 10.3389/fgene.2018.00031.

Caplow, T. (1984): Rule enforcement without visible means: Christmas gift giving in Middletown. In: American Journal of Sociology 89(6): 1306-1323.

Carrier, J. (1991): Gifts, commodities, and social relations: A Maussian view of exchange. In: Sociological Forum 6(1): 119-136.

Clayton, E. W./McGuire, A. L. (2012): The legal risks of returning results of genomics research. Genetics in medicine. In: Official journal of the American College of Medical Genetics 14(4): 473-477.

Cukier, K./Mayer-Schönberger, V. (2013): The rise of big data: How it's changing the way we think about the world. In: Foreign Aff. 92: 28.

DER = Deutscher Ethikrat (2017): Big Data und Gesundheit-Datensouveränität als informationelle Freiheitsgestaltung. Stellungnahme vom 30.11.2017. Unter: https://www.ethikrat.org/fileadmin/ Publikationen/Stellungnahmen/deutsch/stellungnahme-big-data-und-gesundheit.pdf [08.02 2021].

Dixon, P./Gellman, R. (2014): The scoring of America: How secret consumer scores threaten your privacy and your future. In: World Privacy Forum, Online-Publikation 02.04.2014. Unter: https:// www.ftc.gov/system/files/documents/public_comments/2014/08/00014-92369.pdf [25.02.2021].

Dove, E. S. et al. (2012): Power to the people: A wiki-governance model for biobanks. In: Genome Biology 13: 158, Online-Publikation 29.05.2012. DOI: 10.1186/gb-2012-13-5-158.

El-Sayed, S./Prainsack, B. (2021): Blue chips and white collars: Whose data science is it? In: Harvard Data Science Review, Online-Publikation 29.01.2021. DOI: 10.1162/99608f92.ea29ee5a. 
Erlich, Y./Narayanan, A. (2014): Routes for breaching and protecting genetic privacy. In: Nat Rev Genet 15(6): 409-421.

EURAT = Ethische und rechtliche Aspekte der Totalsequenzierung des menschlichen Genoms (2015): Stellungnahme: Eckpunkte für eine Heidelberger Praxis der Ganzgenomsequenzierung. Unter: http://www.uni-heidelberg.de/md/totalsequenzierung/mk_eurat_journal_d_2016_web.pdf [27.06.2017].

Favaretto, M. et al. (2020): What is your definition of big data? Researchers' understanding of the phenomenon of the decade. In: PloS one 15(2): e0228987, Online-Publikation 25.02.2020. DOI: 10.1371/ journal.pone.0228987.

FAZ = Frankfurter Allgemeine Zeitung (2018): Krebsforschung lässt Bürger mitreden. Neuer Patientenbeirat. 24.11.2018. Unter: https://www.faz.net/aktuell/wissen/krebsforschung-schafft-patientenbeirat-und-laesst-buerger-mitreden-15899242.html [25.02.2021].

Frydman, G. (2010): A patient-centric definition of participatory medicine. Unter: https://participatorymedicine.org/epatients/2010/04/a-patient-centric-definition-of-participatory-medicine. html [25.02.2021].

Geissler, J. et al. (2017): Improving patient involvement in medicines research and development: A practical roadmap. In: Therapeutic innovation \& regulatory science 51(5): 612-619.

Gymrek, M. et al. (2013): Identifying personal genomes by surname inference. In: Science 339(6117): 321-324.

Hughes, B. et al. (2008): Health 2.0 and medicine 2.0: Tensions and controversies in the field. In: J Med Internet Res 10(3): e23, Online-Publikation 06.08.2008. DOI: 10.2196/jmir.1056.

Hulsen, T. et al. (2019): From big data to precision medicine. In: Frontiers in medicine 6: 34, OnlinePublikation 01.03.2019. DOI: 10.3389/fmed.2019.00034.

Hummel, P. et al. (2019): Data donations as exercises of sovereignty. In: Krutzinna, J./Floridi, L. (Hrsg.): The ethics of medical data donation. Philosophical Studies Series 137. Springer, Cham: 23-54.

Hummel, P. et al. (2021): Data sovereignty: A review. In: Big Data \& Society 8(1): p.2053951720982012, Online-Publikation 22.01.2021. DOI: 10.1177/2053951720982012.

Husedzinovic, A. et al. (2015): Stakeholders' perspectives on biobank-based genomic research: systematic review of the literature. In: Eur J Hum Genet 23(12): 1607-1614.

Joly, Y. et al. (2013): Genetic discrimination and life insurance: a systematic review of the evidence. In: BMC Medicine 11(1): 25, Online-Publikation 31.01.2013. DOI: 10.1186/1741-7015-11-25.

Jungkunz, M. et al. (im Druck): Haben Patient*innen die Pflicht, klinische Daten für die Sekundärnutzung in der Forschung bereitzustellen? In: Ethik in der Medizin (eingereicht).

Jungkunz, M et al. (2021): Secondary use of clinical data in data-gathering, non-interventional research or learning activities: definition, types, and a framework for risk assessment. In: JMIR 23(6): e26631. DOI: $10.2196 / 26631$.

Kant, I. (1785/1984): Grundlegung zur Metaphysik der Sitten. Reclam, Stuttgart.

Kant, I. (1797/2017): Die Metaphysik der Sitten. Suhrkamp, Frankfurt am Main. 
Kaye J, et al. (2015): Dynamic consent: A patient interface for twenty-first century research networks. In: European Journal of Human Genetics 23: 141-146.

Kirschsieper, E. M. (2016): Datensouveränität im digitalen Zeitalter. In: Friedrichsen M./Bisa, P. J. (Hrsg.): Digitale Souveränität. Springer VS, Wiesbaden: 237-246.

Kitchin, R./McArdle, G. (2016): What makes big data, big data? Exploring the ontological characteristics of 26 datasets. In: Big Data \& Society 3(1): p.2053951716631130, Online-Publikation 01.06.2016. DOI:10.1177/2053951716631130.

Knoppers, B. M. (2014): Framework for responsible sharing of genomic and health-related data. In: Hugo J 8(1): 3, Online-Publikation 17.10.2014. DOI: 10.1186/s11568-014-0003-1.

Knoppers, B. M./Thorogood, A. M. (2017): Ethics and big data in health. In: Current Opinion in Systems Biology 4: 53-57.

Krutzinna, J./Floridi, L. (Hrsg.) (2019): The ethics of medical data donation. Philosophical Studies Series 137. Springer, Cham.

Laney, D. (2001): 3D data management: Controlling data volume, velocity and variety. Meta-Group Research Note 06.02.2001. Unter: https://community.aiim.org/blogs/doug-laney/2012/08/25/dejavvvu-gartners-original-volume-velocity-variety-definition-of-big-data [03.03.2021].

Mayer-Schönberger, V./Cukier, K. (2013): Big data: A revolution that will transform how we live, work and think. John Murray Publishers, London.

McKinsey Global Institute (2011): Big data: The next frontier for innovation, competition, and productivity. Unter: https://www.mckinsey.com/ /media/McKinsey/Business\%20Functions/ McKinsey\%20Digital/Our\%20Insights/Big\%20data\%20The\%20next\%20frontier\%20for\%20 innovation/MGI_big_data_exec_summary.pdf [25.02.2021].

McMahon, A. et al. (2020): Big data governance needs more collective responsibility: the role of harm mitigation in the governance of data use in medicine and beyond. In: Medical law review 28(1): 155-182.

NAS = National Academies of Science [National Research Council [US] Committee on A Framework for Developing a New Taxonomy of Disease] (2011): Toward precision medicine: Building a knowledge network for biomedical research and a new taxonomy of disease. National Academies Press, Washington.

Ossorio, P. (2012): Taking aims seriously: repository research and limits on the duty to return individual research findings. Genetics in medicine. In: Official Journal of the American College of Medical Genetics 14(4): 461-466.

Paradise, J. et al. (2008): Managing incidental findings in human subjects research: analysis and recommendations. In: J Law Med Ethics 36(2): 219-248.

Prainsack, B. (2017a): Personalized medicine: empowered patients in the 21st century? New York University Press, New York.

Prainsack, B. (2017b): Research for personalised medicine: Time for solidarity. In: Medicine and Law 36(1): 87-98. 
Prainsack, B. (2019): Data donation: How to resist the iLeviathan. In: Krutzinna, J./Floridi, L. (Hrsg.): The ethics of medical data donation. Philosophical Studies Series 137. Springer, Cham: 9-22.

Prainsack, B. (2020): The political economy of digital data: introduction to the special issue. In: Policy Studies 41(5): 439-446.

Prainsack, B./Buyx, A. (2017): Solidarity in biomedicine and beyond. Cambridge University Press, Cambridge.

Price, W. N./Cohen, I. G. (2019): Privacy in the age of medical big data. In: Nature medicine 25(1): 37-43. Pullman, D. et al. (2012): Personal privacy, public benefits, and biobanks: a conjoint analysis of policy priorities and public perceptions. In: Genet Med 14(2): 229-235.

Rawls, J. (1979): Eine Theorie der Gerechtigkeit. Suhrkamp, Frankfurt am Main.

Schleidgen, S. et al. (2017): Applying systems biology to biomedical research and health care: a précising definition of systems medicine. In: BMC Health Serv Res 17: 761, Online-Publikation 21.11.2017. DOI: 10.1186/s12913-017-2688-z.

Schleidgen, S. et al. (2019): Between minimal and greater than minimal risk: How research participants and oncologists assess data-sharing and the risk of re-identification in genomic research. In: Philosophy \& Technology 32(1): 39-55.

Sharon, T. (2016): The googlization of health research: from disruptive innovation to disruptive ethics. In: Personalized Medicine 13(6): 563-574.

Simon, J./Rieder, G. (2017): Big data: A new empiricism and its epistemic and socio-political consequences. In: Pietsch, W. et al. (Hrsg.): Berechenbarkeit der Welt? Philosophie und Wissenschaft im Zeitalter von Big Data. Springer VS, Wiesbaden: 85-105.

Singer, P. (1972): Famine, affluence, and morality. In: Philosophy \& Public Affairs 1(3): 229-243.

Spahl, W./Prainsack, B. (2021): Lived solidarity in the Austrian healthcare system. In: EASST Review 40(1). Unter: https://easst.net/article/lived-solidarity-in-the-austrian-healthcare-system/ [06.05.2021].

Spitz, M. et al. (2021): Rechtlicher Rahmen für eine privilegierte Nutzung klinischer Daten zu Forschungszwecken. In: MedR 39: 499-504. DOI: 10.1007/s00350-021-5898-7.

Supple, D. et al. (2015): From tokenism to meaningful engagement: best practices in patient involvement in an EU project. In: Research Involvement and Engagement 1: 5, Online-Publikation 25.06.2015. DOI: 10.1186/s40900-015-0004-9.

Thorogood, A. et al. (2019): Return of individual genomic research results: are laws and policies keeping step? In: European Journal of Human Genetics 27(4): 535-546.

Tkacz, N. (2015): Wikipedia and the politics of openness. University of Chicago Press, Chicago.

Vogt, H. et al. (2016): The new holism: P4 systems medicine and the medicalization of health and life itself. In: Medicine, Health Care and Philosophy 19(2): 307-323.

Voigt, T. H. et al. (2020): Willingness to donate genomic and other medical data: results from Germany. In: European Journal of Human Genetics 28(8): 1000-1009.

WMA = World Medical Association (2013): WMA Declaration of Helsinki - Ethical Principles for Medical Research Involving Human Subjects. In: JAMA 310(20): 2191-2194. 
Wolf, S. M. (2013): Return of individual research results and incidental findings: Facing the challenges of translational science. In: Annual Review of Genomics and Human Genetics 14(1): 557-577.

Wolf, S. M. et al. (2008): Managing incidental findings in human subjects research: analysis and recommendations. In: J Law Med Ethics 36(2): 219-48.

Zawati, M. H./Knoppers, B. M. (2012): International normative perspectives on the return of individual research results and incidental findings in genomic biobanks. In: Genet Med 14(4): 484-489.

Zeitlyn, D. (2003): Gift economies in the development of open source software: anthropological reflections. In: Research Policy 32(7): 1287-1291. 